\title{
A Contrastive Study of Generic Integrity in the Use of Attitudinal Evaluation in Research Articles Written for Different Audiences
}

\begin{abstract}
In today's competitive world of academia, besides offering innovative and robust results, writing scholars must strategically deploy attitudinal evaluation to convince editors and reviewers that their research is valuable and worth publishing. Yet the use of these rhetorical resources can vary across different disciplines, languages and cultures. In addition, the audience for which authors are writing their research (local or international) can significantly influence the way attitudinal evaluation is used.

My corpus consists of 72 research articles (RAs) published internationally in English in three different disciplines (Applied Linguistics, Business Management and Food Technology). A parallel corpus of 36 RAs published locally in Spanish in the same three disciplines has been used as a control group with the aim of establishing whether their different cultures/languages and the different degrees of competitiveness can determine the way attitudinal markers are used.

Manual and electronic analyses have been combined to identify and quantify attitudinal markers in the texts. These markers were classified according to several parameters such as the entity evaluated (Thetela 1997), the type of value expressed and the subject receiving the evaluation. The results for the two sub-corpora were then statistically treated to allow us to find patterns through quantitative contrastive analysis.

The results have shown that, besides significant disciplinary variation in the amount of attitudinal markers used, RA authors use evaluative strategies differently depending on the context of publication. Promoting the significance of one's work seems to be a more important strategy in order to get it published internationally, specially within the most competitive and urban disciplinary fields. Despite being generally regarded as belonging to the same genre, locally published RAs clearly deviate from international RAs in the use of these features, which suggests it may constitute a different subgenre with its own generic integrity. ${ }^{1}$
\end{abstract}

Key words

English for Academic Purposes (EAP); research articles; intercultural rhetoric; variation across disciplines; attitudinal markers 


\section{Introduction}

The publication of a researcher's work is the fundamental means for obtaining professional recognition and prestige and the driving force underlying any scientific endeavour (Becher 1989). However, due to the huge amount of research which is being carried out, getting one's work published, especially in high impact publications, has become increasingly difficult. As a result, apart from producing high quality work, it is also necessary for researchers to dexterously use linguistic rhetorical strategies in order to persuade their audience about the value and originality of their work. One of the main ways in which research writers promote their work is by expressing their personal attitudes and judgements in order to appropriately highlight the value and significance of their work in relation to existing research. This growing use of evaluative and persuasive language in academic writing could indicate that an incipient process of hybridisation is taking place in academic RAs, which increasingly display a blend of interpersonal and rhetorical promotional elements that were generally ascribed to genres connected to the area of advertising. This process involves the colonization of certain academic and professional genres by promotional features resulting in the emergence of mixed or embedded genres (Bhatia 2004).

A considerable number of studies have paid attention to attitudinal evaluation in research articles (see for example Thetela 1997; Hyland 1999, 2005; Shaw 2003; Koutsantoni 2004; Afros and Schryer 2009; and Mur-Dueñas 2010) and research article abstracts (Stotesbury 2003; Martín-Martín and Burgess 2004). More recently, some research has also focused on evaluation in other academic genres such as referee reports (Fortanet 2007), book reviews (Moreno and Suárez 2008; Lorés-Sanz 2009) and oral academic discourse (Swales and Burke 2003; Crawford-Camiciottoli 2004).

Nevertheless, relatively few studies have examined the way attitudinal evaluation is conveyed in RAs across different disciplines and cultures. Moreover, some of the existing studies have only dealt with specific evaluative items, like for example evaluative adjectives (Soler 2002; Swales and Burke 2003) and evaluative that constructions (Hyland and Tse 2005). As a result, there is still a need for quantitative and qualitative data on the frequency, distribution and the linguistic items preferred in different disciplines in order to construct evaluation in RAs, as well as on the impact which different cultural and social contexts can have on the way evaluative resources are used in these texts.

The main objective of the present research work is to contrastively examine the use of attitudinal evaluation in two corpora of RAs written in three different disciplines and published in two different writing cultures and languages: the Anglo-American English-speaking culture, where RAs are written for an international readership, and the Peninsular Spanish culture, were research is directed to a local (Spanish) audience. In doing so, it attempts to establish whether RAs written for a local audience in certain disciplines are guided by the same social 
and epistemological norms and expectations as those published internationally, or whether these local texts deviate from international RAs in the use of these evaluative resources, thus constituting a different subgenre with its own generic integrity.

More specifically, by adopting a double contrastive perspective this research work intends to (1) identify the main differences in the frequency and type of evaluation present in RAs belonging to different disciplines and published internationally, (2) verify whether there exist similar differences among RAs in the same disciplines but written for a local audience and in a different language, (3) to investigate epistemological, ideological and social aspects which can help account for the expected differences across disciplines, languages or contexts of publication and (4) to demonstrate that corpus analysis may be a useful tool in order to identify and account for evaluation in written academic texts.

\section{The concept of evaluation}

A number of concepts including attitude, evaluation, stance, appraisal or affect have been used in the literature in order to roughly describe the way writers project themselves in their texts in order to express their evaluation about the entities they are referring to. In previous works, evaluation has been defined as "anything which indicates the writer's attitude to the value of an entity in the text" (Hunston 1993: 58). In relation to this definition of the term, many authors have described evaluation as a broader concept comprising a number of other interpersonal meanings including epistemic modality (Hunston 1993, Thetela 1997, Thompson and Hunston 2001), moral judgement (Martin 2001), expectedness (Thomson and Hunston 2001) or even self-mention (Hyland 2005).

In this paper, the term attitudinal evaluation (henceforth also evaluation) will be used to refer to expressions conveying the writer's attitudes or feelings as well as value judgements towards a given entity. My definition of attitudinal evaluation coincides with Conrad and Biber's (2001) notion of attitudinal stance, inasmuch as it comprises both the expression of affect (i.e. personal feelings and emotions) and the evaluation of an entity in relation to a certain set of values. As Hunston $(1993,1994)$ suggests, in some genres this assessment of value is made in terms of personal judgement. However, in scientific writing, and more particularly in research articles, the values on which this assessment is based are not personal, but are bonded to the value system of the writer's discourse community. In her model, Hunston establishes three types of evaluation: evaluation of status, value and relevance. The study of attitudinal markers undertaken here however will comprise only evaluation of the value (example 1) and evaluation of the relevance (example 2) of an entity, whereas the evaluation of status (example 3), which roughly corresponds to the epistemic value the author places on an utterance, will be entirely left out. 
(1) This low base rate is likely one reason we failed to find relationships between IM use in the role-play and other variables. (ENG BM4)

(2) Four important factors emerge from these studies that inform the present study. (ENG AL6)

(3) The growth depression and reduced food intake in the group consuming CPI may also be related to the excess of sulphur-containing amino acids. (ENG FTECH17)

\section{Identifying attitude markers in the text}

Arguably, the biggest problem which the study of attitudinal expressions poses for the discourse analyst is finding expedient criteria for identifying attitudinal evaluation in the text. As contended by Hunston $(1993,1994)$ evaluation in academic texts can be implicit and sometimes it may be difficult to identify merely by looking at surface and explicit lexical realizations. In addition, the perception of goodness or badness of an activity depends on the specific social goals of that activity as well as on the particular value system or ideology of the discourse community (Hunston 1993).

Nevertheless, the possible subtle nature of attitudinal evaluation in texts does not need to be an obstacle for the use of corpus analysis. As Hunston herself (1993: 58) and Thetela (1997: 115) have recognised, RAs represent a relatively uniform ideology, which entails that writers of RAs can choose from a rather limited set of semantic values. This hypothesis is somewhat supported by the fact that several researchers have successfully classified the evaluative expressions found in academic texts into a limited set of semantic categories (see Hunston 1993, Thetela 1997, Swales and Burke 2003, among others). It could therefore be argued that, to a large extent, attitudinal expressions could be identified by the lay discourse analyst simply through familiarity with the common value system of RAs. Moreover, as suggested by Stotesbury (2003: 339), evaluation is not only expressed implicitly, but is often rendered explicit by means of conventional evaluative lexis.

The present research will try to show that attitudinal language in RAs is to some extent predictable and identifiable at a lexical level using corpus analysis, as long as it is complemented by manual contextual analysis. Admittedly, such methodology will have limitations as it can overlook some of the linguistic forms as well as certain textual and interpersonal processes and relations which are enacted in the text through evaluation. In spite of these possible inadequacies, it should be stressed that the objective of the present study is not to produce an all-embracing account of attitudinal evaluation in RAs but to compare the use of the most representative forms of this type of evaluation at a lexical level across different disciplines and cultures. Moreover, I agree with Channel (2001) that the 
study of the attitudinal function of a set of words based on concordance examples taken from a corpus can provide useful quantitative data and can help us draw more solid conclusions than those based solely on the intuitions an analyst can form from qualitative analysis of a small number of texts.

\section{Corpus and methodology}

For this analysis two sets of comparable corpora have been used. The first corpus of 72 journal articles comprised three sub-corpora of 24 RAs from the same number of disciplines: Food Technology (FTech), Business Management (BM) and Applied Linguistics (AL). The choice of these three disciplines was determined by the desire to examine widely different areas, i.e. the humanities, the social sciences and the applied sciences. To ensure comparability within this corpus, all the articles were research reports which were randomly selected from recent issues of three high-impact international journals published in English in each of the fields. This corpus was contrasted against a second corpus of $36 \mathrm{RAs}$ from the same three disciplines, all written in Spanish and for a more local audience (see table 1). All the articles were published between 2001 and 2005.

Table 1. The comparable corpora

\begin{tabular}{|l|l|l|}
\hline \multirow{2}{*}{$\begin{array}{l}\text { Corpus ENG } \\
\text { (international publications) }\end{array}$} & 24 RAs in Food Technology & 81,599 words \\
\cline { 2 - 3 } & 24 RAs in Business Management & 146,043 words \\
\cline { 2 - 3 } & 24 RAs in Applied Linguistics & 185,161 words \\
\hline \multirow{2}{*}{$\begin{array}{l}\text { Corpus SP } \\
\text { (local Spanish publications) }\end{array}$} & 12 RAs in Food Technology & 31,032 words \\
\cline { 2 - 3 } & 12 RAs in Business Management & 67,902 words \\
\cline { 2 - 3 } & 12 RAs in Applied Linguistics & 79,928 words \\
\hline TOTAL & 108 RAS & 591,665 words \\
\hline
\end{tabular}

In keeping with the reasoning presented above, the first step in my methodology was to identify a number of lexical items (adjectives, adverbs, nouns and verbs) which were likely to be used with an evaluative or affective meaning in academic texts. Even though several prior lists of this type of lexical items in English (Conrad and Biber 2001; Hyland 1999, 2002; Swales and Burke 2003) were used as a starting point, a final list was elaborated after manual reading of a pilot subcorpus of 6 RAs belonging to each of the disciplines and languages. Each of the tokens in the list of attitude markers was then inserted in the concordance software (WordSmith Tools $4 \AA$ ) to produce raw data and the resulting concordances were analysed in context in order to ascertain their attitudinal value. The tokens that did not contribute to projecting the writer's attitude, as well as all the tokens inserted in literal quotes or in examples were left out.

Inspection of the initial concordance output revealed additional lexical items which were incorporated to the final list and subsequently used to produce new concordances. This whole procedure guaranteed that my analysis would be, at 
least to a large extent, corpus driven, which allowed the examination of the patterns of use of a wide range of evaluative resources across the four disciplines in the corpus.

In order to provide a finer picture of evaluation in RAs, every concordance example was labelled according to several criteria including the type of attitude or value being expressed: emotion, value or relevance and the type of entity analysed. The manual analysis of the concordance data revealed that authors often used attitudinal evaluation to refer to methodological procedures and techniques and that, despite also being linked to the research sphere, evaluation oriented to methods could constitute a separate category in the analysis. A division was therefore made between research-oriented evaluation (including references to findings, results, contributions, etc.) and evaluation oriented towards methods.

Furthermore, during the analysis of the data it became apparent that a broad range of evaluated entities were being included under the category of ROE. Therefore, research-oriented attitudinal markers were further classified taking into account who was the subject being evaluated: (1) the author and his/her research findings, contributions or data; (2) the work of other authors or (3) research issues or approaches in the discipline that had been the focus of, or should be paid attention by the research literature. By doing this, it was expected that the data obtained would more accurately account for the way attitudinal meaning was used to negotiate the relationship between the writer and her readership.

\section{Results and discussion}

\section{Overall disciplinary variation in the two corpora}

As described in the previous section, electronic and manual analyses were combined to obtain the following results. The two soft knowledge disciplines in the ENG corpus showed the highest incidence of attitudinal evaluation (table 2). Business Management was clearly identified as the discipline showing the highest use of attitudinal markers with 102.2 tokens per 10,000 words, which is over twice as many as in Applied Linguistics (49.9). The lowest frequency was found in Food Technology with only 35.5 markers per 10,000 words and just 14.1 per article. These preliminary results seem to support the assumption that evaluative and affective expressions are circumvented in the technical sciences in favour of a more impersonal and allegedly objective style.

The overall results found in the RAs in the SP corpus are notably different (table 2). The total number of markers per article is highest in AL (41.1), followed by BM, with 29.1 markers per article. Nevertheless, if we consider normalised results by number of words the picture is even more surprising, as the relative number of attitude markers is highest in FTech (65.7), closely followed by AL (61.7). These results would appear to break off RAs in FTech written in Spanish from the conventionalised image of impersonality and objectivity discussed above and would 
support the hypothesis that articles written locally in Spanish in this discipline constitute a somewhat different subgenre with a particular generic integrity.

Table 2. Number of attitudinal markers per article and per 10,000 words in the two corpora

\begin{tabular}{|l|c|c|c|c|c|c|}
\hline \multirow{2}{*}{} & \multicolumn{2}{|c|}{ Applied Linguistics } & \multicolumn{2}{c|}{ Food Technology } & \multicolumn{2}{c|}{ Business Management } \\
\cline { 2 - 7 } & Per article & $\begin{array}{c}\text { Per 10,000 } \\
\text { words }\end{array}$ & Per article & $\begin{array}{c}\text { Per 10,000 } \\
\text { words }\end{array}$ & $\begin{array}{c}\text { Per } \\
\text { article }\end{array}$ & $\begin{array}{c}\text { Per 10,000 } \\
\text { words }\end{array}$ \\
\hline ENG Corpus & 37.8 & 49.0 & 14.1 & 35.5 & 62.2 & 102.2 \\
\hline SP Corpus & 41.1 & 61.7 & 17.0 & 65.7 & 29.1 & 51.4 \\
\hline
\end{tabular}

To my knowledge, in the literature there are few quantitative cross-disciplinary studies of attitudinal markers in RAs, which makes it very difficult to compare these data with previous results. In his study of RA abstracts, Stotesbury (2003) reported that abstracts written in the humanities and social sciences showed a higher use of evaluative attributes (both adjectives and nouns) than those written in the natural sciences. In addition, Hyland $(1999,2004)$ analysed the incidence of attitudinal markers across a corpus of 8 different disciplines and found a considerably more frequent use of these markers in all the soft knowledge disciplines than in the hard knowledge disciplines. Within the former, Hyland reported a higher incidence in the humanities (Applied Linguistics and Philosophy) than in the social sciences (Sociology and Marketing), a finding which is not supported by my results.

Some research has been done on the existing differences in the expression of attitudinal and evaluative meaning across different cultures. Yet this research has focused on a number of different academic genres, which makes it difficult to compare their results to reach clear conclusions. Mur-Dueñas (2010) analysed attitudinal expressions in BM RAs written in Spanish (by native Spaniards) and in English (by American-based scholars) and found that both the frequency of attitude markers and the relative percentage of research oriented attitudinal lexis were similar in the two sub-corpora. Giannoni (2005) used a corpus of RA Discussion sections extracted from Italian and Anglo-Saxon journals in the field of linguistics focusing on the use of negative evaluation. Although his approach was chiefly qualitative, Giannoni found that negative evaluation was almost three times greater in English Discussions, which he related to the tendency of smaller academic languages to show a less assertive stance, with a preference towards collaboration instead of competition. Similar studies on the use of critical acts across Spanish and English book reviews have also reported a substantially higher frequency of these strategies in the English texts (Moreno and Suarez 2008, Lorés-Sanz 2009), which seems to support the hypothesis that there is less competition within smaller, more local academic cultures.

Blagojević (2009) has also contrasted cross-culturally the use of attitudinal expressions in RAs written by Serbian and English authors in three soft-knowledge disciplines: sociology, social psychology and philosophy. However, with 
a few exceptions she focused on markers of affect and emotion, rather than value. This would explain why according to her data the frequency of these markers was higher within the articles written by Serbian authors. Unlike assessments of value and significance, the expression of personal attitude may be more acceptable within more localised or rural cultures, where shared values and collaboration might be prioritised over competition.

The results discussed so far only take into account the overall use of attitudinal markers in the different disciplines and cultures. Yet, in order to provide a more revealing picture of how attitudinal evaluation is used in these texts we need to take into account other factors which shape the interpersonal dynamics of these markers, such as the entity being evaluated (topic-oriented, research-oriented or method-oriented), the type of value assigned to those entities (relevance, value or emotion) and the person (self or others) to which that entity is attached. These aspects will be analysed in the following subsections.

\section{Evaluation oriented to topics, research and methods}

Most studies of evaluation have concerned themselves with issues including the identification and classification of evaluative language, the explanation of how evaluation is constructed in context or the suitability of corpus analysis for the study of this aspect of language. Nevertheless, to my knowledge Thetela (1997) was the first to propose a straightforward criterion for the classification of evaluative language according to the different entities being evaluated. In her work Thetela argues that, when examining evaluation in academic writing, we should take into account not only the values ascribed but also the entities which are being evaluated. Following this premise, she divides evaluative language into researchoriented evaluation (ROE) and topic-oriented evaluation (TOE), where the term "topic" refers to the area which is under investigation in the real world sphere, instead of the investigation itself.

The main reason for using this division lies in that, while in ROE the writer and the reader engage into an interpersonal negotiation about how to judge the different research entities (methods, data or hypotheses), TOE works at a much more localised level and often does not represent the type of strategic dialogue which comes to our mind when discussing evaluation. The following examples are intended to show the existing distinction between research and topic-oriented evaluation:

(4) Adding water during cooking has been observed to decrease palatability and increase cooking losses (Cline et al., 1930) and the flavor of braised veal chops has been reported to decrease with the amount of water added (Lowe et al., 1952), indicating the amount of water added is important to flavor intensity (ENG FTECH13)

(5) From these results, it is likely that in a competitive and uncertain environ- 
ment, a high level of strategic applications contributes more to an increase in performance than in a less uncertain or stable environment. (ENG BM16)

(6) First, this study contributes to the literature by explicitly considering the interaction between applicant race, rater race, and panel racial composition in a structured panel interview. (ENG BM8)

The first two examples illustrate the way topic-oriented evaluation is used. In example 4, the amount of water added to a dish is identified as an important factor when we cook food, thus deserving the attention of research in food technology. Similarly, in example 5, having "a high level of strategic applications" is evaluated as a desirable characteristic of companies in a particular environment. In both cases the evaluated entities do not belong to the research sphere but to the broad areas in which investigation takes place or even to other somewhat related areas. In contrast, example 6 is a case in point of the way a writer assesses the significance of her own research, by highlighting that it provides some new knowledge to the discipline. It could therefore be argued that in this type of evaluation, unlike in the first two examples, writers seek to interact with their readers and persuade them to interpret their work in a particular way, thus facilitating its acceptance for publication.

All tokens of attitudinal evaluation found in the corpus were classified according to the type of entity which was being evaluated. For the sake of providing a finer description, a decision was made to classify references to methodological procedures and techniques as a third type of entity different from ROE and TOE. The results obtained after classifying attitudinal markers into these three categories show several significant patterns of cross-disciplinary variation. As shown below (table 3), in the three disciplines in the ENG corpus evaluation is more frequently topic-oriented than research-oriented. Attitudinal evaluation of research-oriented entities (i.e. findings, results, contributions or limitations of someone's research) was the second most common category also in all three disciplines. Finally, the evaluation of methodological approaches, techniques, procedures or instruments for research, which is also related to the research sphere, was the least frequent.

Table 3. Number of attitudinal markers per article and per 10,000 words (Corpus ENG)

\begin{tabular}{|l|c|c|c|c|c|c|}
\hline & \multicolumn{2}{|c|}{$\begin{array}{c}\text { Applied } \\
\text { Linguistics }\end{array}$} & \multicolumn{2}{c|}{$\begin{array}{c}\text { Food } \\
\text { Technology }\end{array}$} & \multicolumn{2}{c|}{$\begin{array}{c}\text { Business } \\
\text { Management }\end{array}$} \\
\hline & $\begin{array}{c}\text { Per } \\
\text { article }\end{array}$ & $\begin{array}{c}\text { Per 10,000 } \\
\text { words }\end{array}$ & $\begin{array}{c}\text { Per } \\
\text { article }\end{array}$ & $\begin{array}{c}\text { Per 10,000 } \\
\text { words }\end{array}$ & $\begin{array}{c}\text { Per } \\
\text { article }\end{array}$ & $\begin{array}{c}\text { Per 10,000 } \\
\text { words }\end{array}$ \\
\hline $\begin{array}{l}\text { Topic-oriented } \\
\text { (TOE) }\end{array}$ & 20.9 & 27.1 & 7.2 & 21.2 & 32.8 & 54.0 \\
\hline $\begin{array}{l}\text { Research-oriented } \\
\text { (ROE) }\end{array}$ & 11.2 & 14.5 & 3.4 & 9.9 & 19.8 & 32.5 \\
\hline Methods-oriented & 5.7 & 7.4 & 1.5 & 4.4 & 9.6 & 15.8 \\
\hline Total markers & 37.8 & 49.0 & 14.1 & 35.5 & 62.2 & 102.2 \\
\hline
\end{tabular}


Table 4. Number of attitudinal markers per article and per 10,000 words (Corpus SP)

\begin{tabular}{|l|c|c|c|c|c|c|}
\hline \multirow{2}{*}{} & \multicolumn{2}{|c|}{$\begin{array}{c}\text { Applied } \\
\text { Linguistics }\end{array}$} & \multicolumn{2}{c|}{$\begin{array}{c}\text { Food } \\
\text { Technology }\end{array}$} & \multicolumn{2}{c|}{$\begin{array}{c}\text { Business } \\
\text { Management }\end{array}$} \\
\cline { 2 - 7 } & $\begin{array}{c}\text { Per } \\
\text { article }\end{array}$ & $\begin{array}{c}\text { Per 10,000 } \\
\text { words }\end{array}$ & $\begin{array}{c}\text { Per } \\
\text { article }\end{array}$ & $\begin{array}{c}\text { Per 10,000 } \\
\text { words }\end{array}$ & $\begin{array}{c}\text { Per } \\
\text { article }\end{array}$ & $\begin{array}{c}\text { Per 10,000 } \\
\text { words }\end{array}$ \\
\hline $\begin{array}{l}\text { Topic-oriented } \\
\text { (TOE) }\end{array}$ & 26.1 & 39.2 & 9.5 & 36.7 & 9.4 & 16.6 \\
\hline $\begin{array}{l}\text { Research-oriented } \\
\text { (ROE) }\end{array}$ & 10.2 & 15.3 & 3.1 & 11.9 & 13.6 & 24.0 \\
\hline Methods-oriented & 4.8 & 7.3 & 4.4 & 17.1 & 6.1 & 10.8 \\
\hline Total markers & 41.1 & 61.7 & 17.0 & 65.7 & 29.1 & 51.4 \\
\hline
\end{tabular}

Table 5. Relative incidence (\%) of the evaluated entities across disciplines and cultures

\begin{tabular}{|l|c|c|c|c|c|c|}
\hline & \multicolumn{2}{|c|}{$\begin{array}{c}\text { Applied } \\
\text { Linguistics }\end{array}$} & \multicolumn{2}{c|}{$\begin{array}{c}\text { Food } \\
\text { Technology }\end{array}$} & \multicolumn{2}{c|}{$\begin{array}{c}\text { Business } \\
\text { Management }\end{array}$} \\
\hline & ENG & SP & ENG & SP & ENG & SP \\
\hline $\begin{array}{l}\text { Topic-oriented } \\
\text { (TOE) }\end{array}$ & $55.2 \%$ & $63.5 \%$ & $59.7 \%$ & $55.9 \%$ & $52.8 \%$ & $32.4 \%$ \\
\hline $\begin{array}{l}\text { Research-oriented } \\
\text { (ROE) }\end{array}$ & $29.7 \%$ & $24.7 \%$ & $27.9 \%$ & $18.14 \%$ & $31.7 \%$ & $46.7 \%$ \\
\hline Methods-oriented & $15.1 \%$ & $11.8 \%$ & $12.4 \%$ & $26.0 \%$ & $15.5 \%$ & $20.9 \%$ \\
\hline
\end{tabular}

Authors writing in English in AL and FTech devote a rather similar percentage of their attitude markers (table 5) to evaluating research-oriented and topic-oriented entities, while evaluation of methods is only slightly less frequently chosen in FTech (12.4\%) than in AL (15.1\%). In BM the relative incidence of attitude markers referring to research $(31.7 \%)$ and to methods $(15.5 \%)$ was only a bit higher than in the other two disciplines. In brief, the overall allocation (\%) of attitude markers to each of the three entities is rather similar in all the disciplines under analysis. This may indicate that, despite different disciplinary expectations as regards the extent to which promotional strategies are acceptable, authors share similar rhetorical goals and priorities when it comes to deciding which aspects must be evaluated. In relation to this, it could also be hypothesized that cultural and contextual aspects (impact, competition, etc.) sometimes have more bearing on the use of certain rhetorical resources than disciplinary idiosyncrasies. In addition, the very high overall number of attitudinal markers in BM, together with the high proportion of markers oriented to research entities and methods, suggests BM is a highly competitive discipline where the appropriate interpersonal negotiation of the value and significance of one's research (in relation to that of others) is required when drafting one's text for publication.

The incidence of the three types of evaluated entities in the disciplines in the local Spanish RAs (SP corpus) shows somewhat different patterns (table 4). The frequency of attitudinal markers in the Spanish AL articles is rather similar to that found in RAs written in English for an international audience, both in global 
terms and when considering the type of entity under evaluation, perhaps with the only exception of TOEs, which are somewhat more frequent in Spanish.

The Spanish FTech corpus had an overall frequency of attitudinal markers per article (17) which is close to the number found in the English comparable FTech corpus (14.1). Yet, FTech articles in Spanish were typically much shorter than those in English, which means that Spanish articles were much more densely evaluated per 10,000 (65.7 tokens, compared to 35.5). In particular, attitudinal markers were far more common in the Spanish RAs when referring to methodological aspects, which could be possibly explained by the fact that researchers publishing locally may be using less standardised procedures and must therefore make a bigger rhetorical effort to convince readers of the viability and soundness of their methods. This discrepancy could also be accounted for by the fact that, due to the standardization of the methodological procedures found in RAs published in international journals, their description is made in a highly concise, almost perfunctory way, allowing little room for strategic evaluative resources.

Even larger cultural differences were found in the use of attitudinal markers across the two sets of articles from BM. One major area of discrepancy was overall frequency: attitudinal markers were only half as frequent in Spanish local RAs as in English international RAs. In addition, the relative percentage of attitudinal evaluation aimed at each of the different entities (table 5) was remarkably different in the Spanish BM corpus, where only $32 \%$ of all the evaluative markers made reference to entities outside the research sphere (TOEs), compared to $52.8 \%$ in the ENG corpus. These differences in the evaluation of entities inside and outside the research sphere can be explained by the different degree of competition present in each context of publication. Research writers publishing internationally must necessarily stress the centrality for the discipline of the issues and topics they focus on in their research in order to show that this research constitutes a significant contribution to the field and therefore deserves publication. Conversely, competition for publication is not as strong in local Spanish publications, which means that claiming centrality is not so essential in order to get one's work published locally.

\section{Parameters of attitudinal evaluation}

Another aspect which has been taken into account in this study is the parameter of value or semantic category of evaluation which is preferred across the disciplines and cultures in the two corpora. As discussed earlier, aside from emotion, attitudinal evaluation in RAs can be classified into a limited number of semantic values consistent with the goal of the text and the value systems of the disciplinary community (Hunston 1993, 1994; Thetela 1997). In this analysis these parameters of value were subsumed into three major semantic categories of evaluative meaning: assessment, usefulness and significance. By evaluation of assessment we mean judgements of value made along a good-bad scale which typically refer to the soundness, complexity, breadth or worth of the entity analysed (example 7). The 
second type of value analysed, usefulness, refers to the degree of efficacy or utility of the research entity analysed in terms of how well it helps to achieve certain goals (example 8). Finally, evaluation of significance has to do with highlighting the originality, novelty of an entity, which is in fact a measure of its importance for the discipline (example 9).

(7) Se trata de dos propuestas estrechamente relacionadas y coherentes entre sí, puesto que Kathpalia realizó su investigación bajo la dirección de Bhatia. (SP AL14)

These two proposals are closely linked and are mutually coherent, inasmuch as Kathpalia did her research under Bhatia's supervision. (author's translation)

(8) Keeney did not develop specific measures of these constructs but his proposed means and fundamental objectives provided a useful base for item generation and instrument development. (ENG BM11)

(9) Our results give new insights into the possible mechanisms causing the storage-induced firmness of carrot. (ENG FTECH12)

The set of values which RA authors use when evaluating research entities is closely linked to the main rhetorical goal of the research article, i.e. persuading the audience about the originality, soundness and significance of the work being reported (Latour and Wolgar 1979). The achievement of these goals usually entails a particular interpersonal negotiation between the writer and her audience, since the former needs to evaluate her own research in connection to that of others so as to persuade her audience to recognise the contributions she has made and to accept the claims she is presenting. Nevertheless, this kind of interaction does not take place when entities outside of the research sphere are being assessed, as the kind of evaluation made is potentially less controversial and often is unrelated to the main rhetorical goal of the RA. In sum, topic-oriented evaluation does not involve the same type of strategic and interpersonal negotiation and is not steered by the same type of values as research-oriented evaluation, which is why for the analysis of the different parameters of value used in the two corpora I have focused only on attitudinal markers evaluating a research entity.

Table 6. Relative incidence per 10,000 words of the different parameters of attitudinal evaluation

\begin{tabular}{|l|c|c|c|c|c|c|}
\hline & \multicolumn{2}{|c|}{ Applied Linguistics } & \multicolumn{2}{c|}{ Food Technology } & \multicolumn{2}{c|}{ Business Management } \\
\hline & ENG & SP & ENG & SP & ENG & SP \\
\hline Assessment & 2.81 & 3.00 & 3.80 & 1.93 & 15.54 & 11.19 \\
\hline Significance & 9.40 & 8.38 & 4.90 & 8.38 & 13.35 & 10.90 \\
\hline Usefulness & 1.08 & 3.00 & 0.61 & 1.61 & 2.67 & 1.47 \\
\hline Emotion & 1.19 & 0.88 & 0.61 & 0.00 & 0.89 & 0.44 \\
\hline Total & 14.47 & 15.26 & 9.93 & 11.92 & 32.46 & 24.01 \\
\hline
\end{tabular}


As the results indicate (table 6), there were quite notable differences across disciplines in the type of parameter of value which was preferred when referring to research, while these differences were somewhat less relevant across languages. The evaluations made in terms of significance (+ significant / - significant) were the most common in Applied Linguistics and Food Technology in both the SP and the ENG corpus. The distribution was rather different in Business Management, where markers of assessment were roughly as frequent as markers of significance in the SP corpus, and proportionally more frequent in the ENG corpus. Expressions of usefulness also appeared consistently in all the corpora. These expressions were clearly less common than markers of assessment or relevance in all the articles except for the Spanish RAs in FTech and AL, where their frequency was almost equivalent to that of assessment markers. Finally, expressions of personal emotion were quite rare across all the corpora, which indicates that their use is clearly restricted by the expectations of impersonality and objectivity which are typical of scientific writing. Even though their low incidence make cross-cultural differences irrelevant, it is surprising that these expressions were less frequent in the RAs written in Spanish. This discrepancy may be due to the fact that the linguistic exponents for expressing emotion which appeared in the corpora (surprising(ly)/sorprendente(mente), curiously/ curiosamente, etc.) are altogether quite uncommon in Spanish.

To summarise, even though there are substantial differences in the amount of evaluative markers which are used across languages and contexts of publication, the parameters of value or semantic categories of evaluation which are preferred are similar in RAs written in Spanish and English in the same field. This suggests that the social and epistemological norms and expectations present in each field of knowledge have a great impact on the type of values which disciplinary members are required to use as point of reference for their evaluations.

\section{Subject receiving attitudinal evaluation}

In this last section of the study the description of attitudinal evaluation will be narrowed down by examining who receives or is referred to when expressing attitudinal meaning in RAs. As shown above, attitudinal evaluation oriented to research entities and methods constitutes a potent interpersonal device for the negotiation of the readers' acceptance and recognition for the claims presented by the author of a RA. Nevertheless, the pragmatic function and intended effects of these evaluative acts are obviously very different depending on who the target of the evaluation is. Following this rationale, all markers of attitudinal evaluation that referred to methods or research entities were classified into three categories: (1) research studies, data or methods belonging to the author of the RA (labelled as Research1, Datal or Methods1, respectively), (2) research studies or methods belonging to other specific authors in the discipline (Research2 or Methods2) and (3) general concepts or methods which are often used or referred to in the discipline $($ Research $(x)$ or Methods $(x))$. 
Table 7. Relative percentage of attitudinal evaluation aimed at a different subject

\begin{tabular}{|l|c|c|c|c|c|c|}
\hline & \multicolumn{2}{|c|}{$\begin{array}{c}\text { Applied } \\
\text { Linguistics }\end{array}$} & \multicolumn{2}{c|}{$\begin{array}{c}\text { Food } \\
\text { Technology }\end{array}$} & \multicolumn{2}{c|}{$\begin{array}{c}\text { Business } \\
\text { Management }\end{array}$} \\
\hline & ENG & SP & ENG & SP & ENG & SP \\
\hline Research1 & $49.8 \%$ & $38.5 \%$ & $60.5 \%$ & $29.7 \%$ & $66.9 \%$ & $35.6 \%$ \\
\hline Research(Data1) & $20.1 \%$ & $15.6 \%$ & $16.0 \%$ & $29.7 \%$ & $6.1 \%$ & $25.8 \%$ \\
\hline Research2 & $18.6 \%$ & $26.2 \%$ & $2.5 \%$ & $8.1 \%$ & $9.1 \%$ & $9.2 \%$ \\
\hline Research(x) & $11.5 \%$ & $19.7 \%$ & $21.0 \%$ & $32.4 \%$ & $17.9 \%$ & $29.4 \%$ \\
\hline Total per 10,000 words & 14.47 & 15.26 & 9.93 & 11.92 & 32.46 & 24.01 \\
\hline Method1 & $54.7 \%$ & $65.5 \%$ & $75.0 \%$ & $71.7 \%$ & $74.5 \%$ & $68.5 \%$ \\
\hline Method2 & $5.1 \%$ & $8.6 \%$ & $0.0 \%$ & $9.4 \%$ & $4.8 \%$ & $5.5 \%$ \\
\hline Method(x) & $39.4 \%$ & $25.9 \%$ & $25.0 \%$ & $18.9 \%$ & $16.5 \%$ & $26.0 \%$ \\
\hline Total per 10,000 words & 7.4 & 7.3 & 4.4 & 17.1 & 15.8 & 10.8 \\
\hline
\end{tabular}

The results obtained show that in general the majority of the markers of attitudinal evaluation were used by RA writers in all the disciplines in order to evaluate their own study or their own methodology, which allowed them to establish the value or significance of their contribution and the soundness of the methodological procedures they used. It is very significant to note that in every discipline the percentage of ROEs which referred to the author's own study (Research 1) was always higher in the RAs written in English for an international audience than in those written for a local Spanish audience. In particular, the percentage of Research1 attitudinal markers was roughly twice as high in FTech and BM articles in the ENG corpus (60.5\% and $66.9 \%$ respectively) as in the comparable SP corpora $(29.7 \%$ and $35.6 \%)$. These findings confirm the hypothesis that contextual factors such as size of the academic community and, even more importantly, competition for publication, have a great bearing on the relative propensity of RA authors to focus their evaluative acts on their own research study.

In addition, the results indicate that the urge to promote one's research through evaluation is not as high in Applied Linguistics, which may be explained by the fact that is a less competitive, more "rural" (Becher 1989) community. The small differences found in the relative incidence of attitudinal markers in AL articles written in English and Spanish also supports the hypothesis that assessing the relevance of one's work is not as important in this discipline, as the pressure to establish the novelty of one's contribution is less intense than in other academic fields.

It must also be noted that RAs in the two languages showed a similar tendency to focus on one's work when evaluating methods. This is not surprising however, inasmuch as the need to evaluate one's methods is not only related to the degree of competition for publication that exists in a discipline, but also to the degree of consensus in the use of certain methodological techniques. This consensus may be lower in smaller academic communities, where methods may not need to be as clearly agreed upon as in larger, more competitive communities. 


\section{Conclusions}

The results presented in this study have shown relevant differences in the amount of attitudinal evaluation which is used in RAs published internationally in different disciplines. Frequent attitudinal evaluation seemed to be required in BM articles, while evaluation was less frequent in AL articles and was very restricted in similar articles in FTech. These discrepancies must be related to the different epistemological norms and social expectations which obtain in each disciplinary field.

It was also found that some of these differences are arbitrated by the culture where the articles were produced and by the size of the audience for which they were drafted. For instance, attitudinal markers were quite more often used in FTech RAs written in Spanish than in those RAs written in English. Conversely, attitudinal markers were roughly twice as frequent in BM articles in the ENG corpus as in the SP corpus, a finding which was related to the higher competition found for international publication. These apparently contradictory data can be partly explained once the analysis of evaluation is enhanced in order to take into account key aspects such as the entity under evaluation or the subject receiving the evaluation. Thus, the huge increase in the use of evaluative markers in FTech articles written in Spanish can be explained by the finding that most of this increment was restricted to topic-oriented evaluations (which by their own nature are not designed to promote the value of the author's work), and to method-oriented evaluation (which can be related to the lower degree of consensus with regards to methods we find in smaller local disciplinary communities). These differences in the amount of evaluation needed in RAs written locally and internationally support the hypothesis that RAs written in Spanish for local audiences lack integrity with certain disciplinary conventions present in international articles.

Notwithstanding these cross-disciplinary differences in the relative frequency of attitude markers, the results showed that RA authors from different disciplines allocate a similar percentage of their evaluative resources to the three entities for evaluation identified in this study: research, methods and topics. This finding suggests that members of the academia share somewhat similar rhetorical goals and priorities when it comes to deciding which aspects must be evaluated.

Disciplinary differences in the parameters of value preferred for expressing attitudinal evaluation were also worth noting. Evaluation of significance was prioritised in AL articles in both languages, while evaluation in terms of assessment was preferred in BM articles in the two languages. There were however similarities in the categories of evaluation which are preferred within each field, indicating that these parameters are to some extent determined by the social and epistemological norms and expectations of each discipline.

Finally, my results have revealed an inclination by authors in FTECH and BM to focus expressions of evaluation on one's work ( $60.5 \%$ and $66.9 \%$ respectively) 
and to relate it to the work of other specific researchers (Research2), as opposed to the more "diffused evaluation" (Giannoni 2005) present in AL RAs. This preference for more "targeted" (Giannoni 2005) evaluation can be related to the necessity to produce an assertive stance due to the stronger competition present in urban communities ${ }^{2}$ (Becher 1989) such as BM and FTECH. In contrast, in more rural, less competitive communities like Applied Linguistics RA writers do not need to promote their work to the same extent.

To summarise, it has been argued that, besides disciplinary variations in the amount of attitudinal markers used, evaluation works differently depending on the size of the academic community and the existing degree of competition. In international RAs attitudinal evaluation focuses to a greater extent on stressing the value of one's work than it does in local Spanish RAs. This difference is more apparent in the more urban and competitive cultures like BM and FTech, where contextual differences (impact, competition, rewards, etc.) lead writers to be more assertive and promotional when addressing an international audience. Based on these findings it could be argued that Spanish RAs published locally in these fields use attitudinal evaluation to a different extent, as well as to refer to different entities and subjects and that it is sometimes used in order to perform a different rhetorical function. In that respect, these RAs constitute a separate subgenre with a different generic integrity. Consequently, RA writers publishing in the local Spanish context and hoping to publish their work internationally should be aware of the peculiarities in the use of interpersonal resources in that context.

In my view, this study helps to support the claim that Corpus Linguistics is a valid tool for examining evaluation. It also shows that attitudinal evaluation is open to quantification from a lexical perspective and that it can be better accounted for if our analysis takes into account several aspects such as the type of value and entity under analysis, and the subject receiving the evaluation.

Corpus studies like the one presented here can provide valuable data for novice writers by identifying the preferred expressions and resources for performing certain rhetorical moves involving attitudinal evaluation. In addition, they can be helpful for EAP instructors in order to teach future writers a range of acceptable structures for highlighting the usefulness, soundness or novelty of their research, as well as the importance or value of other aspects and entities under study in their disciplines.

Future research into attitudinal evaluation across cultures and disciplines should analyse intra-lingual variation within a discipline to determine homogeneity in the use of these practices. In addition, a focus on evaluation in specific sections and moves within the research article would provide a finer picture of the interpersonal and pragmatic interactions which take place in the text. Future studies should also undertake the design of pedagogical materials based on data describing preferred expressions for the expression of particular evaluative acts in each discipline. Finally, researchers should investigate the best pedagogical tools to enable EAP instructors to efficiently teach these aspects to novice writers and practitioners in general. 


\section{Notes}

1 The present research has been developed within the research group InterLAE (funded by Gobierno de Aragón) and as part of the research project "La integridad genérica en la comunicación académica y profesional: los géneros y su correlación con las prácticas discursivas y con la cultura de distintas comunidades profesionales (FFI2009-09792)", funded by the Spanish Ministerio de Ciencia e Innovación (MICIIN).

According to Becher (1989), "urban" communities are those where members are closely connected and recent developments in the area impact other researchers quickly. Typically, pressure to publish is great. In contrast, "rural" communities show lower consensus, that is, less secure criteria with regards to research problems to be addressed and the methodological procedures to be followed. Research in rural communities tends to be local and has a rather delayed impact on other members of the group. Moreover, competition for publication is usually smaller.

\section{References}

Afros, Elena and Catherine Schryer (2009) 'Promotional (meta)discourse in research articles in language a literary studies'. English for Specific Purposes 28, 58-68.

Becher, Tony (1989) Academic Tribes and Territories: Intellectual Inquiry and the Cultures of Disciplines. Milton Keynes: SRHE/Open University Press.

Bhatia, Vijay K. (2004) Worlds of Written Discourse: A Genre-Based View. London and New York: Continuum.

Biber, Douglas and Edward Finegan (1989) 'Styles of stance in English: Lexical and grammatical marking of evidentiality and affect'. Text 9 (1), 93-124.

Blagojević, Savka (2009) 'Expressing attitudes in academic research articles written by English and Serbian authors'. Linguistics and Literature 7 (1), 63-73

Channel, Joanna (2001) 'Corpus-based analysis of evaluative lexis'. In: Hunston, Susan and Geoff Thomson (eds.) Evaluation in Text: Authorial Stance and the Construction of Discourse. Oxford, New York: Oxford University Press, 38-55.

Conrad, Susan and Douglas Biber (2001) 'Adverbial marking of Stance in Speech and Writing'. In: Hunston, Susan and Geoff Thomson (eds.) Evaluation in Text: Authorial Stance and the Construction of Discourse. Oxford, New York: Oxford University Press, 56-73.

Crawford Camiciottoli, Belinda (2004) 'Audience-oriented relevance markers in lectures'. In: Del Lungo, Gabriella and Elena Tognini (eds.) Academic Discourse: New Insights into Evaluation. Bern: Peter Lang, 81-97.

Fortanet, Inmaculada (2007) 'Evaluative language in peer review referee reports'. English for Academic Purposes 7 (1), 27-37.

Giannoni, Davide (2005) 'Negative Evaluation in Academic Discourse. A Comparison of English and Italian Research Articles'. Linguistica e Filologia 20, 71-99.

Hunston, Susan (1993) 'Evaluation and ideology in scientific writing'. In: Ghadessy, Mohsen (ed.) Register Analysis: Theory and Practice. London: Pinter Publishing, 57-73.

Hunston, Susan (1994) 'Evaluation and organization in a sample of written academic discourse'. In: Coulthard, Malcolm (ed.) Advances in Written Text Analysis. London: Routledge, 191-218.

Hyland, Ken (1999) 'Disciplinary discourses: writer stance in research articles'. In: Candlin, Christopher and Ken Hyland (eds.) Writing: Texts, Processes and Practices. London: Longman, 99-121.

Hyland, Ken (2002) 'Academic argument: Induction or interaction'. Revista Canaria de Estudios Ingleses 44, 29-45.

Hyland, Ken (2004) 'Engagement and Disciplinarity: the other side of evaluation'. In: Del Lungo, Gabriella and Elena Tognini (eds.) Academic Discourse: New Insights into Evaluation. Bern: Peter Lang, 13-30. 
Hyland, Ken (2005) Metadiscourse. London: Continuum.

Hyland, Ken and Polly Tse (2005) 'Hooking the reader: a corpus study of evaluative that in abstracts'. English for Specific Purposes 24 (2), 123-139.

Koutsantoni, Dimitra (2004) 'Attitude, certainty and allusions to common knowledge in scientific research articles'. Journal of English for Academic Purposes 3, 163-182.

Martín-Martín, Pedro and Sally Burgess (2004) 'The rhetorical management of academic criticism in research article abstracts'. Text 24, 171-195.

Latour, Bruno, and Steve Wolgar (1979) Laboratory Life: The Social Construction of Scientific Facts. Beverly Hills, Ca.: Sage.

Lorés-Sanz, Rosa (2009) '(Non-) Critical voices in the reviewing of history discourse: A crosscultural study of evaluation'. In: Hyland, Ken and Giuliana Diani (eds.) Academic evaluation: Review genres in university settings. Basingstoke: Palgrave MacMillan, 143-160.

Martin, J. R. (2001) 'Beyond exchange: Appraisal systems in English'. In: Hunston, Susan and Geoff Thomson (eds.) Evaluation in Text: Authorial Stance and the Construction of Discourse. Oxford, New York: Oxford University Press, 142-175.

Moreno, Ana and Lorena Suárez (2008) 'A study of critical attitude across English and Spanish academic book reviews'. Journal of English for Academic Purposes 7, 15-26.

Mur-Dueñas, Pilar (2010) 'Attitude markers in business management research articles: a crosscultural corpus-driven approach'. International Journal of Applied Linguistics 20 (1), 50-72.

Shaw, Philip (2003) 'Evaluation and promotion across languages'. Journal of English for Academic Purposes 2, 343-357.

Soler, Viviana (2002) 'Analysing adjectives in scientific discourse: an exploratory study with educational applications for Spanish speakers at advanced university level'. English for Specific Purposes 21, 145-165.

Stotesbury, Hilkka (2003) 'Evaluation in research article abstracts in the narrative and hard sciences'. Journal of English for Academic Purposes 2, 327-341.

Swales, John and Amy Burke (2003) "IIt's really fascinating work": Differences in evaluative adjectives across academic registers'. 3rd North American Symposium on Corpus Linguistics and Language Teaching. New York: Rodopi, 1-18.

Thetela, Puleng (1997) 'Evaluated entities and parameters of value in academic research articles'. English for Specific Purposes 16, 101-118.

Thompson, Geoff and Susan Hunston (2001) 'Evaluation: An Introduction'. In: Hunston, Susan and Geoff Thomson (eds.) Evaluation in Text: Authorial Stance and the Construction of Discourse. Oxford, New York: Oxford University Press, 1-27.

EnRiQue Lafuente-Millán completed his PhD in English Linguistics at the Universidad Zaragoza (Spain), where he is a Senior Lecturer at the Department of English Studies in the Faculty of Education. His main lines of research include genre analysis, written academic discourse, corpus studies and contrastive studies, both from a disciplinary and cultural perspective. He has published a number of articles on these issues in journals and volumes in the area and has presented his research at several national and international conferences and forums. He is also a member of the InterLAE research group (www.interlae.com). During the first semester of 2012 he was a visiting scholar at Deakin University (Melbourne, Australia) and he is currently doing casual teaching at the School of Languages and Linguistics, the University of Melbourne.

Address: Enrique Lafuente-Millán, Department of Linguistics and Applied Linguistics, School of Languages and Linguistics, The University of Melbourne, VIC 3010, Australia. [e-mail: elafuente@unimelb.edu.au, elafuen@unizar.es] 